\title{
Adding Constraints to an Algorithm for Orthogonal Graph Drawing
}

\author{
Roland Wiese and Michael Kaufmann \\ Universität Tübingen, Wilhelm-Schickard-Institut, Sand 13, 72076 Tübingen, \\ Germany, \\ $\{$ wiese/mk\}@informatik. uni-tuebingen.de
}

There are two kinds of approaches for orthogonal graph drawing: one supports planar and almost planar graphs (Giotto [6], Kandinsky) and is based on a mincost flow algorithm. The second does not take planarity into account. It is therefore conceptually much simpler and runs in linear-time. Representatives of such concepts are the papers 5.32 . In a variant of this last paper, Biedl/Kaufmann 4 achieve the theoretically best area bound. The idea is the following:

The graph is drawn such that each edge bends exactly once. Outgoing edges leave the vertex from the right or left side, incoming edges arrive at the top or bottom side. Edges are directed in a quasi-s-t-ordering such that each vertex gets at least one incoming and one outgoing edge. Then we assign disjoint rows to the vertices such that the number of outgoing edges leaving the vertex $v$ to different directions is nearly balanced. The same is done for the column assignment where the numbers of incoming edges from different sides has to be balanced. Applying this simple scheme yields an area bound of $(m+n) / 2 \times(m+n) / 2$ for the graph.

Making graph algorithms aware of constraints is a very important task. We do it here for the Biedl/Kaufmann scheme.

First we consider the behaviour of the algorithm in respect to the aspect ratio of the drawn nodes. With the original algorithm the aspect ratio of $v$ can get as bad as $2: \operatorname{deg}(v)$ because the number of in-and outgoing edges for each vertex is not balanced. In the orginal paper 4, the authors already gave an idea how to achieve an aspect ratio of 1:2, based on Eulerian paths. Unfortunately, the nice balancing of the edges is given up, such that the area bound is doubled. We improve this scheme so that it works very satisfying in practice.

On the theoretical side, we give improvements for some special cases, namely for graphs with degree at most 3 and for planar graphs. We conjecture that an improvement to $(2 / 3 \cdot m+n / 2) \times(2 / 3 \cdot m+n / 2)$ of the area is possible using the Eulerian path technique in an appropriate way.

In practice a flexible approach based on edge flipping heuristics provides even better aspect ratios and keeps the size of drawing area close to the good bounds of the unconstraint algorithm.

Next, we look at edge constraints, where the edges have to be directed in a certain direction. There is a straightforward approach already published in [1]. Here the row and column assignment in the basic scheme is done such that the 

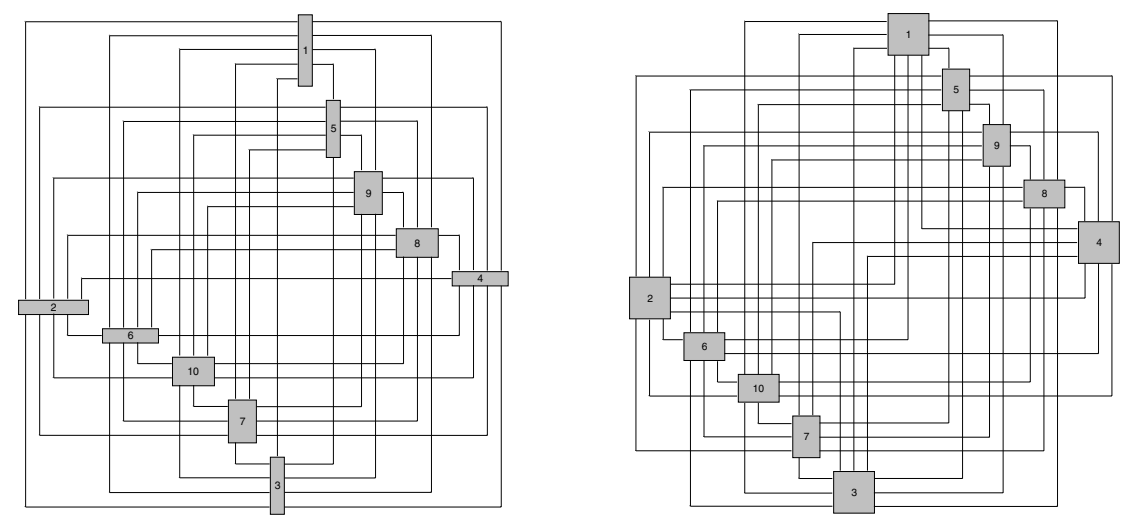

Fig. 1. $K_{10}$ drawn without and with aspect ratio constraints. For the drawing on the left an edge flipping heuristic was applied.

constraints for the edge directions are fulfilled. But again we lose the balancing of the edges so that the area bound is even quadrupled. We propose an alternative approach that works especially good for one-dimensional constraints.
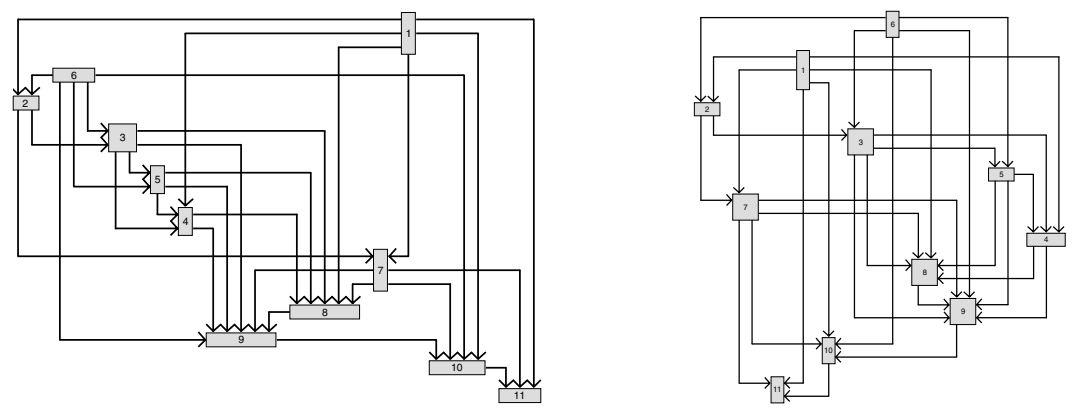

Fig. 2. Two downward drawings of the same graph. On the left drawn with a simple algorithm and on the right with our linear-time constraint algorithm.

\section{References}

1. Biedl, T.C., Orthogonal Graph Drawing: The 3-Phases method, Ph.D.thesis, 1997.

2. Biedl, T.C., B. Madden, I.G. Tollis, The 3-Phase Method: A Unified Approach to Orthogonal Graph Drawing, Proceedings of GD'97, LNCS 1353, 391-403, 1997.

3. Biedl, T.C., G. Kant, A better heuristic for orthogoanl graph drawings. Proceedings of ESA'94, LNCS 855, 24-35, 1994.

4. Biedl, T.C., M. Kaufmann, Area-Efficient Static and Incremental Drawings of HighDegree Graphs. Proceedings of ESA'97, LNCS 1284, 37-52, 1997.

5. Papakostas, A., I.G. Tollis, Orthogonal drawing of high degree graphs with samll area and few bends, Proceedings of WADS'97, LNCS, 1997.

6. Tamassia, R. On Embedding a Graph in the Grid with the Minimum Number of Bends, SIAM Journal of Computing, vol. 16, no. 3, 421-444, 1987. 